\title{
A Propagation Model for Subsurface and Through-Wall Imaging Applications under the Frequency Dispersion Perspective
}

\author{
Ana Vazquez Alejos ${ }^{1}$ and Muhammad Dawood ${ }^{2}$ \\ ${ }^{1}$ Department of Teoria de la Señal y Comunicaciones, University of Vigo, Vigo, 36310 Pontevedra, Spain \\ ${ }^{2}$ Klipsch School of Electrical and Computer Engineering, New Mexico State University, Las Cruces, NM 88003, USA \\ Correspondence should be addressed to Ana Vazquez Alejos; analejos@uvigo.es
}

Received 19 July 2013; Accepted 13 September 2013

Academic Editor: Francesco Soldovieri

Copyright ( 2013 A. Vazquez Alejos and M. Dawood. This is an open access article distributed under the Creative Commons Attribution License, which permits unrestricted use, distribution, and reproduction in any medium, provided the original work is properly cited.

\begin{abstract}
The frequency-dependent behavior in subsurface and through-the-wall media is analyzed in this paper as well as the formation of the Brillouin precursor waveforms inherently related to this feature. The emergence of these forerunners is presented as a plausible form to explain classical impairments observed in imaging technologies. The evolution of mono- and multicycle rectangular and first derivative Gaussian pulses through two dispersive media-concrete blocks and soil-is analyzed using a frequencydomain technique and the Debye dielectric model to characterize the media, at operating frequencies below $3 \mathrm{GHz}$. The frequencydomain approach facilitated to check the influence of some parameters considered critical for the precursor emergence-operating frequency, input pulse configuration, and internal structure of the underlying medium-results in a versatile tool suitable for any kind of modulated input signal propagated through any dispersive medium. The internal multireflection model has been considered as the most suitable model to describe the transmission process underlying both subsurface and through-wall imaging technologies. Two different moisture contents have been considered for concrete as a parameter to determine the performance of through-wall imaging radar from the precursor formation perspective. The results reveal that precursor is a phenomenon to take into account for application demanding larger signal-to-noise ratios.
\end{abstract}

\section{Introduction}

The frequency dependence of the dielectric properties in some materials is an important issue affecting the performance of radar imaging applications mainly in terms of both signal-to-noise ratio and pulse duration alterations. The frequency-dependent features can lead to the appearance of the waves, namely, Brillouin and Sommerfield precursors [14]. Despite precursors being a well-known phenomenon, we usually do not find the imaging and the expected precursor linkage, especially ultrawideband system configurations for dispersive medium.

The main distinguishing properties of the precursor waves have awakened interest for their probable use in different applications in a broad range of frequency bands, such as radio standoff inspection, biosensing, subsurface (underwater/underground) communications or throughwall-tissue imaging [5-9]. For instance, the nonexponential peak amplitude decay of these precursor waves would improve the link range which also means an optimal penetration depth in a given media; walls made of concrete, therefore, present an ideal opportunity to detect and utilize the precursors to obtain high signal-to-noise ratio (SNR) and/or greater penetration depths.

The precursor formation in the transmitted or propagating signal, however, can produce undesired features such as pulse broadening and downshifting of the carrier frequency of the modulated pulses $[1,3]$. The time spreading undergone by the signal through the dispersive media turns frequency dispersion into an extremely important impairment in receiver systems based on correlation processing [3].

Nevertheless, the appearance of the precursors depends on many factors, including the frequency window, enough frequency bandwidth, and the input signal configuration. In any case, neglecting or eliminating this forerunner component removes an important portion of the received signal 


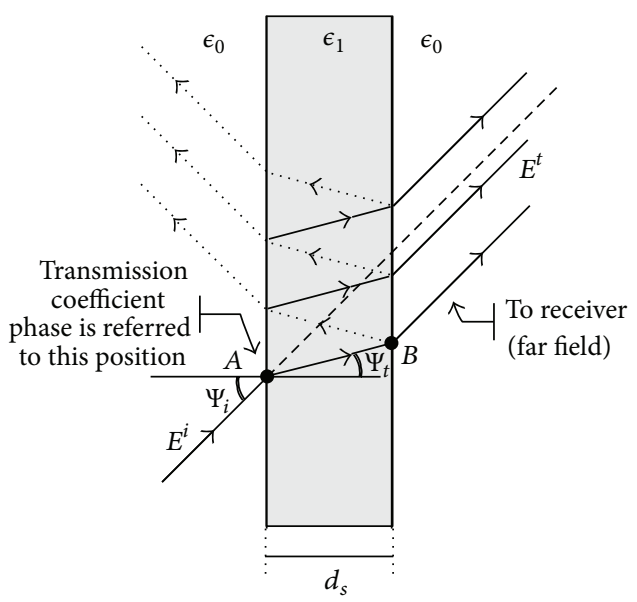

FIGURE 1: IMR transmission model for concrete and soil slabs.

power [4], and this fact can lead to under optimal receiver hardware performance as well as inaccurate inversion methods in imaging applications. The pulse broadening or the algebraically amplitude decay introduced in the received signal can be better understood by an exhaustive analysis of the precursor fields.

For the cases of through-wall imaging (TWI) and underground imaging (UGI), the frequency-dependent attenuation and phase constants of the walls constructed of concrete structures [7-11] can obscure the interior due to reduced SNR.

We apply a frequency-domain method based on considering the frequency response $H(z, f)$ of the medium under study. This response is derived from the theoretically or experimentally achieved propagation constant, and it depends on the material thickness or distance travelled within the medium $z$. The frequency response can be seen as a frequency filter containing information about the effects of attenuation and phase for each frequency component of the potential signal travelling through it. We can then analyze the evolution of any input signal $x(z, t)$ through the dispersive medium for any propagation depth $z[3-6,12-16]$.

Nevertheless, the transmission process undergone by the signal travelling through the material can strongly condition the output. For a medium consisting of slabs of materials rather than half-space medium, usually assumed in theoretical simulations, the double interface or boundary conditions present a finite thickness medium, and the underlying transmission process can give rise to internal successive reflections arising out on the material edges with a more than minor influence on the propagated field.

In order to extract the material frequency response to an incoming EM signal for TWI and UGI, an internal multireflection model is assumed thereby considering the different spurious components due to the internal reflections occurring inside the material considered as a finite slab in the TWI case or as a finite half-space or single slab in the UGI case. For the latter subsurface case, it would be plausible to consider a stack of slabs to model the gradually changing dielectric properties of the soil medium which can be seen

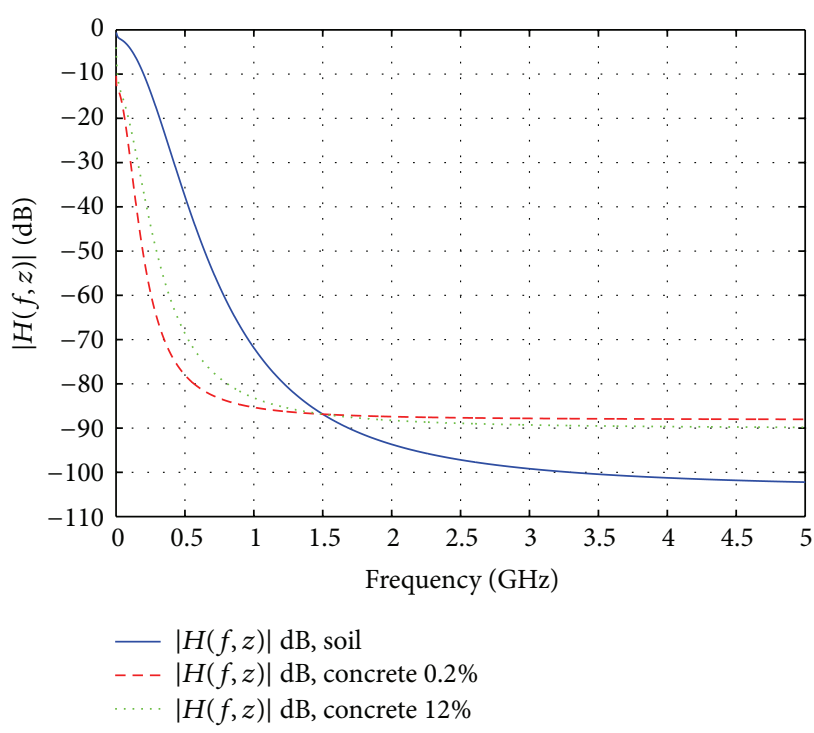

Figure 2: Frequency response assuming: (a) soil and (b) concrete.

as a superposition of different layers, from a superficial cover of turf, following sandy soil and dry soil till deep stratums of rocky soil.

For the purposes of this paper, we considered two pulses: the first derivative of the Gaussian pulse and a rectangular pulse. Monocycle and multicycles settings are presented to better illustrate the precursor performance. The dispersive media consisted of soil and concrete structures characterized by an extended Debye model for frequencies below $3 \mathrm{GHz}$ $[1,10]$.

In Section 2 we introduce the main elements required to perform the analysis of the propagation through a dispersive medium: the theoretical formulation and the dielectric model as well as the frequency transfer of the medium. In Section 3 we present and discuss the results achieved for the simulations done for the soil and concrete models for this study. Finally, the paper is concluded in Section 4.

\section{Theoretical Analysis of Dispersive Propagation}

We assumed a sine-modulated signal $x(t)$ as in (1), with $f_{0}$ being the carrier frequency, and $s(t)$ represents the baseband information-bearing signal of duration $T_{b}$ :

$$
x(z=0, t)=s\left(\frac{t}{T_{b}}\right) \cdot \sin \left(2 \pi f_{0} t\right)
$$

The modulated pulse $x(t)$, or equivalently $x(z=0, t)$, passes through the medium having a frequency response $H(z, f)$, acting as a frequency filter or channel response, as indicated in (2). The final result, $y(z, t)$, transformed to the time domain by an inverse Fourier Transform, is the signal affected by the dispersion phenomenon after travelling 


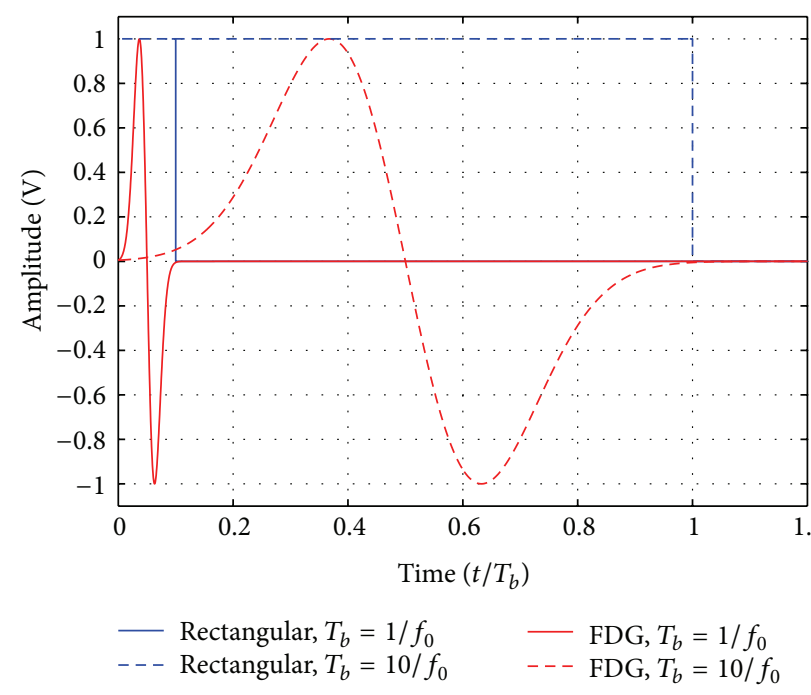

FIGURE 3: Baseband rectangular and FDG pulses in time domain.

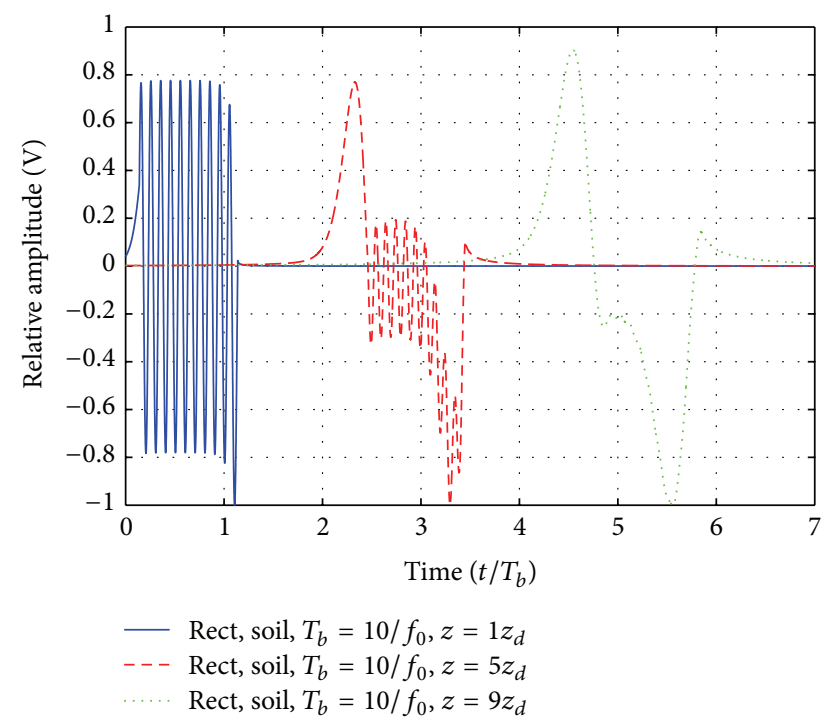

Figure 4: Normalized electric field evolution through different propagation distances within a dielectric slab of soil for a rectangular pulse with $f=1.5 \mathrm{GHz}$ and $T_{b}=10 / f_{0}$.

through the dispersive medium at a distance $z$, as expressed in (3):

$$
\begin{gathered}
Y(z, f)=X(f) \cdot H(z, f), \\
y(z, t)=F^{-1}\{X(z=0, f) \cdot H(z, f)\} .
\end{gathered}
$$

Only a linear polarization is considered for this study. As can be noticed, all parameters controlling the pulse shape and the sinusoidal carrier frequency can easily be changed, within the constraints given by the bandwidth to be covered.

In (2), we observe that each frequency component $X\left(f_{k}\right)$ is differently affected upon propagation through the dispersive material, modeling the behavior of the different spectral components of the input pulse $x(t)$. The material frequency

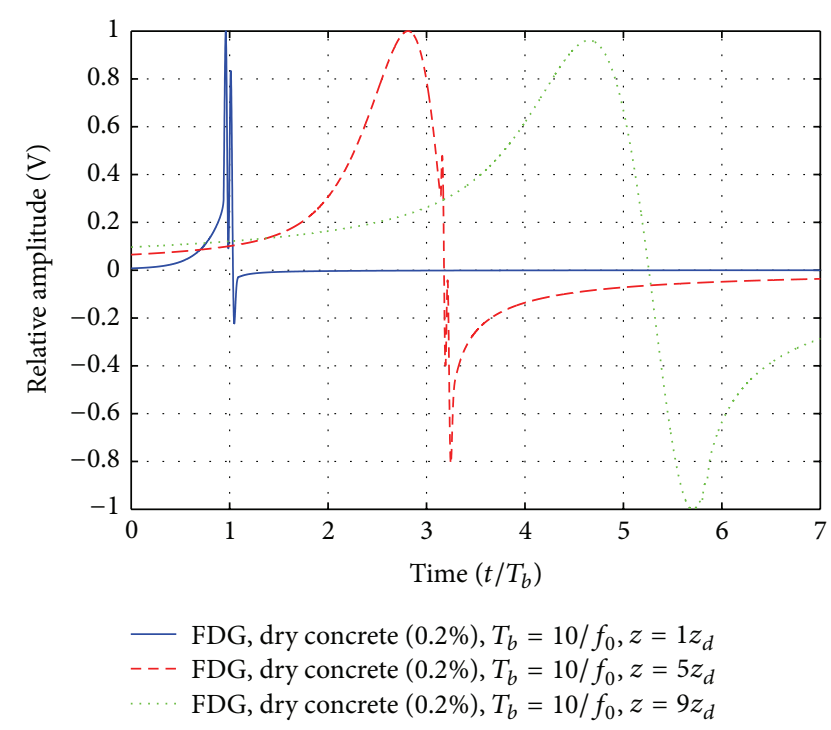

FIGURE 5: Normalized electric field evolution through different propagation distances within a dielectric slab of dry concrete $(0.2 \%$ moisture content) for a FDG pulse with $f=1.5 \mathrm{GHz}$ and $T_{b}=$ $10 / f_{0}$.

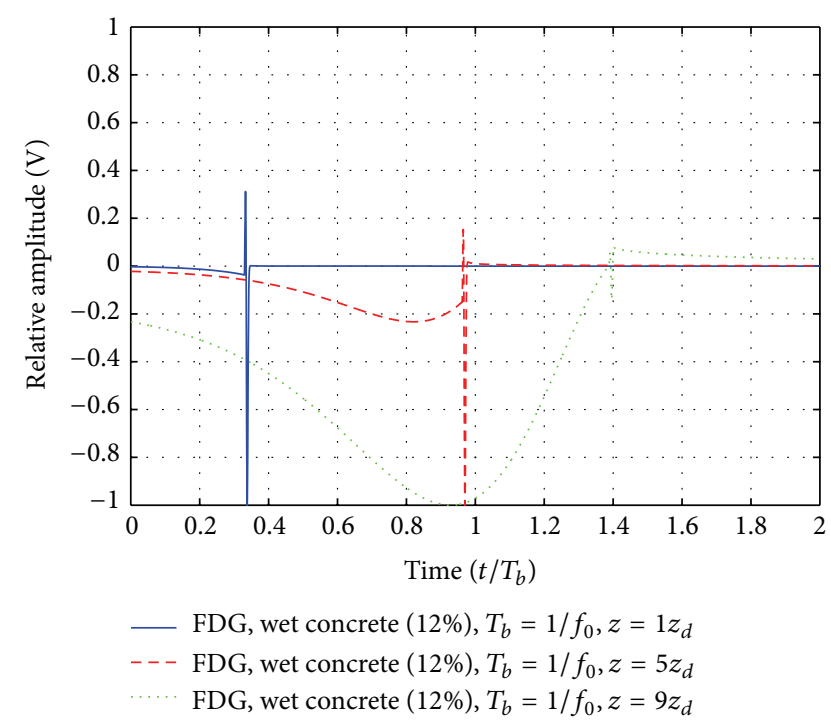

FIGURE 6: Normalized electric field evolution through different propagation distances within a dielectric slab of wet concrete $(12 \%$ moisture content) for a FDG pulse with $f=1.5 \mathrm{GHz}$ and $T_{b}=1 / f_{0}$.

response $H(z, f)$ depends on the transmission process occurring through the medium. Several electromagnetic models have been developed trying to provide a realistic formulation of the transmission process of an EM wave through the material. When the material thickness $d_{m}$ is large compared to the wavelength and/or the material losses are high, the transmission and reflection coefficients, respectively, $T_{g}$ and $R_{g}$ of a plane wave on a dielectric slab can be approximated by the Fresnel's model [17]. For this case, only one ray contribution is ensured to be received. When material thickness is not large enough, the internal successive reflections become 


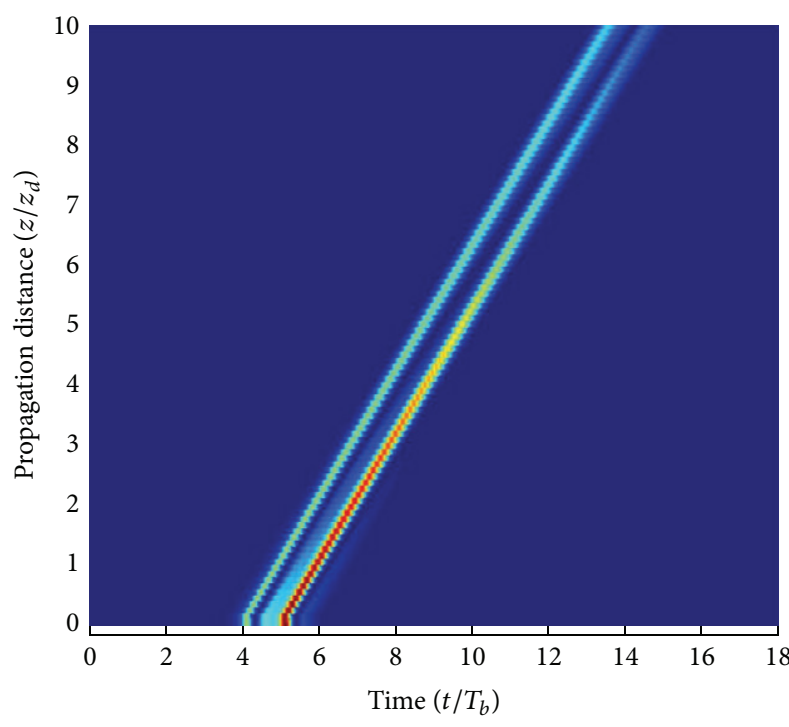

(a)

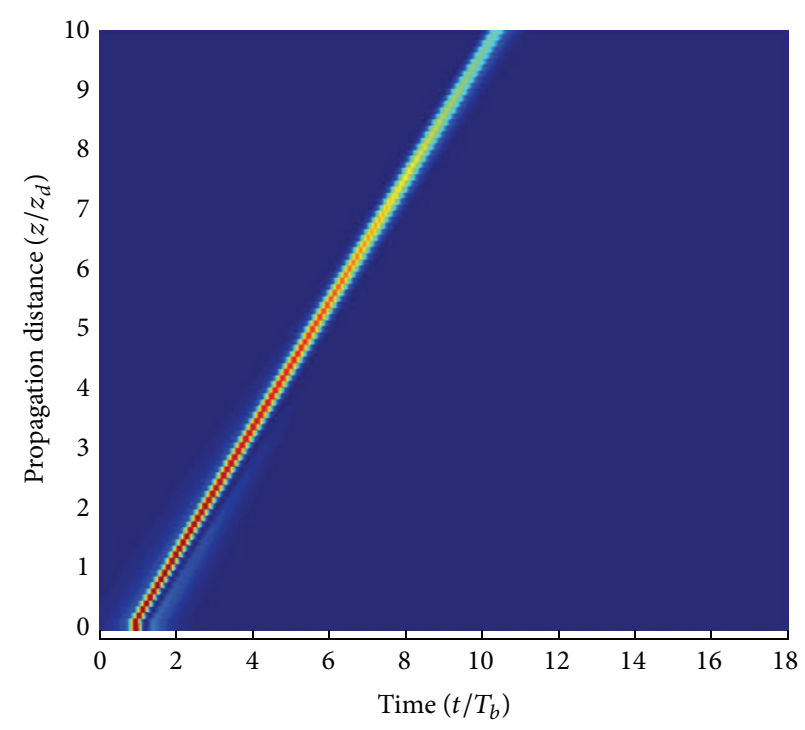

(b)

FIGURE 7: Spatial evolution of the normalized electric field intensity distribution through a dielectric slab of soil at $f_{0}=1.5 \mathrm{GHz}$, IMR model: (a) rectangular pulse with $T_{b}=10 / f_{0}$ and (b) FDG pulse with $T_{b}=1 / f_{0}$. Amplitude levels range in the interval $[0,1]$.

a dominant phenomenon; the internal multireflection model (IMR) [18] seems a more accurate approach of the underlying transmission physics.

The transmission coefficient $T_{g}$ is a frequency-dependent function and can be understood as the material frequency response $H(z, f)$ also representing the boundary conditions due to the medium interfaces (points $A$ and $B$ in Figure 1), as well as the transmission through the medium to reach a given propagation distance $z$. According to the geometry explained in Figure $1, H(z, f)$ can be defined as in (4), analogous to the transmission coefficient $T_{g}$ given for the IMR model [18]:

$$
H(f, z)=\frac{E_{B}}{E_{A}}=\frac{T_{\mathrm{am}} \cdot T_{\mathrm{ma}} \cdot e^{-j \gamma_{m}(f) z}}{1+\Gamma_{\mathrm{am}} \cdot \Gamma_{\mathrm{ma}} \cdot e^{-j 2 \gamma_{m}(f) z}}
$$

with $T_{\mathrm{am}}, T_{\mathrm{ma}}, \Gamma_{\mathrm{am}}$, and $\Gamma_{\mathrm{ma}}$ denoting the Fresnel transmission and reflection coefficients for the air-material (am) and material-air (ma) interfaces, with $T_{i j}=2 \sqrt{\varepsilon_{j}(f)} /\left(\sqrt{\varepsilon_{i}(f)}+\right.$ $\left.\sqrt{\varepsilon_{j}(f)}\right)$ and $T_{i j}=1+\Gamma_{i j}, i, j=a, m$. The value of the propagation constant $\gamma_{m}(f)$ required for (4) is derived from the theoretical $\varepsilon_{r}(f)$ as indicated in

$$
\gamma_{m}(f)=\alpha(f)+j \beta(f)=j \frac{2 \pi f}{c} \sqrt{\varepsilon_{r m}^{\prime}(f)-j \varepsilon_{r m}^{\prime \prime}(f)},
$$

where $\varepsilon_{r m}^{\prime}(f)$ and $\varepsilon_{r m}^{\prime \prime}(f)$ are the real and imaginary parts of the complex relative permittivity, $c$ is the speed of light in free space, and $f$ is the frequency in $\mathrm{Hz}$.

From (2) and (5) we can infer that the assumed dielectric model becomes vital to achieve a correct estimation of signal propagation through the dispersive medium and consequently for an accurate evaluation of the precursor formation, as mentioned in [3-5]. For instance, the relative dielectric permittivity model also gives us an idea of the most suitable frequency window to achieve the formation of the precursors.

We have considered Debye models to characterize the soil and concrete media considered in this study. The complex relative dielectric permittivity for soil $\varepsilon_{r, \text { soil }}(f)$ is given as a Rocard-Powles-Debye model [9] as indicated in

$$
\varepsilon_{r, \text { soil }}(f)=\sum_{j=i=1}^{3} \frac{a_{j}}{\left(1-i \cdot \omega \tau_{j}\right) \cdot\left(1-i \cdot \omega \tau_{f_{j}}\right)}
$$

with constants $a_{1}=13.63, a_{2}=0.33$, and $a_{3}=0.293$; rotational relaxation times $\tau_{1}=1.82 \cdot 10^{-6} \mathrm{~s}, \tau_{2}=1.97 \cdot 10^{-8} \mathrm{~s}$, $\tau_{3}=2.36 \cdot 10^{-10} \mathrm{~s}$; frictional relaxation times $\tau_{f 1}=8.78$. $10^{-11} \mathrm{~s}, \tau_{f 2}=1.12 \cdot 10^{-10} \mathrm{~s}$, and $\tau_{f 3}=1.00 \cdot 10^{-15} \mathrm{~s}$; and with $\omega$ the frequency in $\mathrm{rad} / \mathrm{s}$.

The Debye model for the concrete structures is given in [10] as by (7):

$$
\varepsilon_{r}(\omega)=\varepsilon_{\infty}+\frac{\Delta \varepsilon}{1+j \cdot\left(\omega / \omega_{r}\right)^{2}}-j \cdot \frac{\left(\omega / \omega_{r}\right) \cdot \Delta \varepsilon}{1+\left(\omega / \omega_{r}\right)^{2}}-j \cdot \frac{\sigma_{\mathrm{dc}}}{\omega \varepsilon_{0}},
$$

where $\Delta \varepsilon=\varepsilon_{s}-\varepsilon_{\infty}$ is the difference between the real part of the complex relative permittivity at DC and high frequency $\omega_{r}$ is the relaxation angular frequency, and $\sigma_{\mathrm{dc}}$ is the DC electrical conductivity. The parameter values are summarized in Table 1 according to [10] for two moisture contents, $0.2 \%$ (dry concrete) and $12 \%$ (wet concrete).

In Figure 2 we show the frequency response $H(z, f)$ for the two Debye media, soil, and concrete structures, as a function of the material thickness, or distance travelled within the medium, $z / z_{d}$, normalized to the reference propagation depth $z_{d}=\alpha^{-1}\left(f_{0}\right)$ meters, with $\alpha(f)$, the attenuation constant of the dispersive media; $z_{d}=1.0184 \mathrm{~m}$ (soil), 


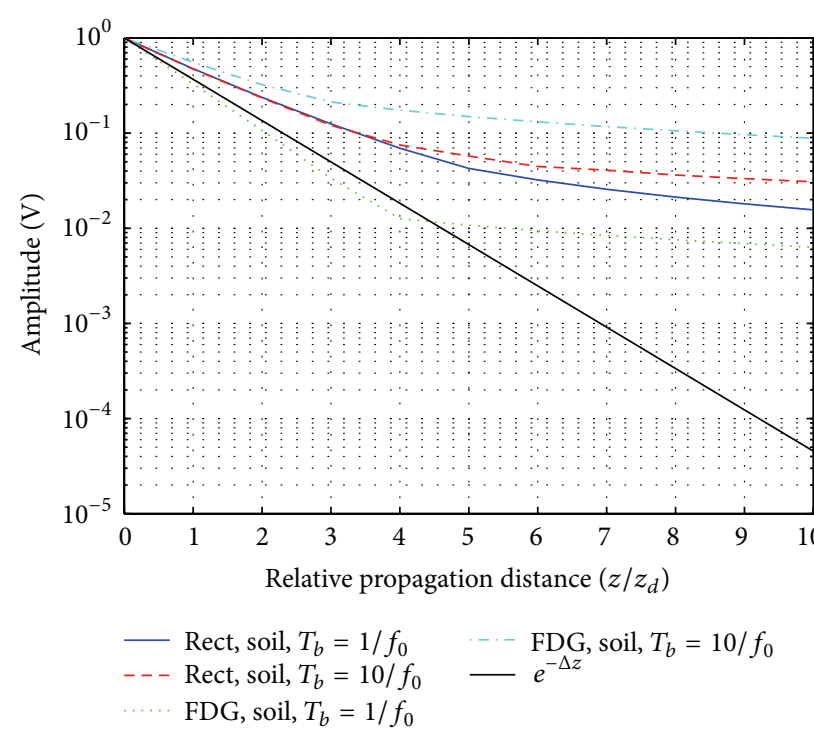

Figure 8: Peak amplitude as a function of propagation distance through soil for rectangular and FDG pulses of duration $T_{b}=10 / f_{0}$ and $T_{b}=1 / f_{0}$ at $f_{0}=1.5 \mathrm{GHz}$.

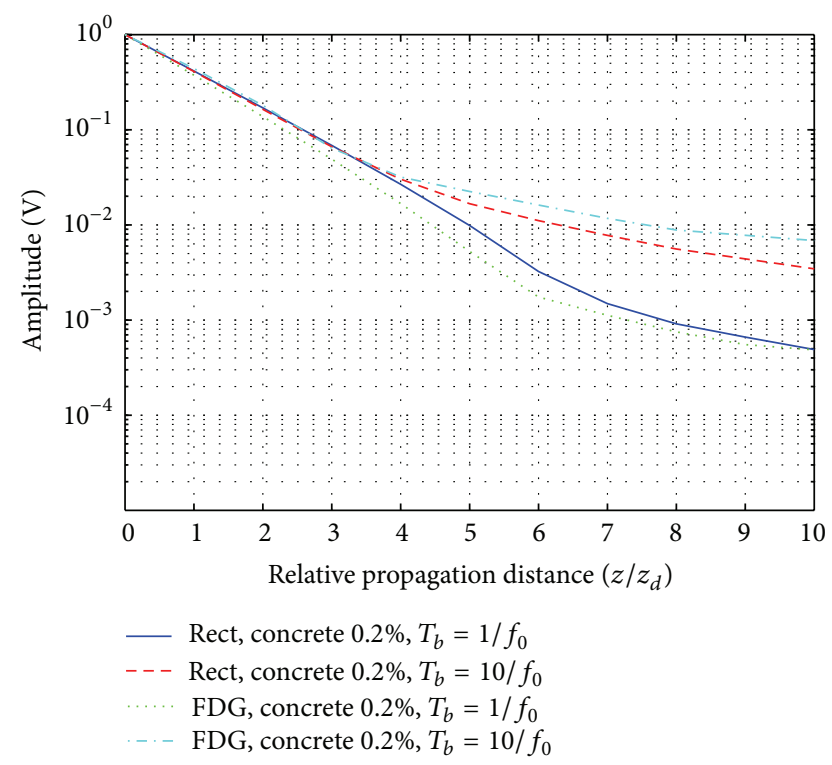

FIGURE 9: Peak amplitude as a function of propagation distance through dry concrete $(0.2 \%$ moisture $)$ for rectangular and FDG pulses of duration $T_{b}=10 / f_{0}$ and $T_{b}=1 / f_{0}$ at $f_{0}=1.5 \mathrm{GHz}$.

TABle 1: Parameters for concrete Debye model as per [10].

\begin{tabular}{lcccc}
\hline Moisture content & $\varepsilon_{s}$ & $\varepsilon_{\infty}$ & $\omega_{r}$ & $\sigma_{\mathrm{dc}}$ \\
\hline $0.2 \%$ & 4.814 & 4.507 & $1.22 \cdot 10^{9}$ & $6.06 \cdot 10^{-4}$ \\
$12 \%$ & 12.84 & 7.42 & $1.64 \cdot 10^{9}$ & $20.6 \cdot 10^{-3}$ \\
\hline
\end{tabular}

$z_{d}=2.9178 \mathrm{~m}$ (dry concrete), and $z_{d}=0.1512 \mathrm{~m}$ (wet concrete). The influence of the moisture content is not a determining parameter of the trend shown by $H(z, f)$, and it mostly affects the lower frequencies.

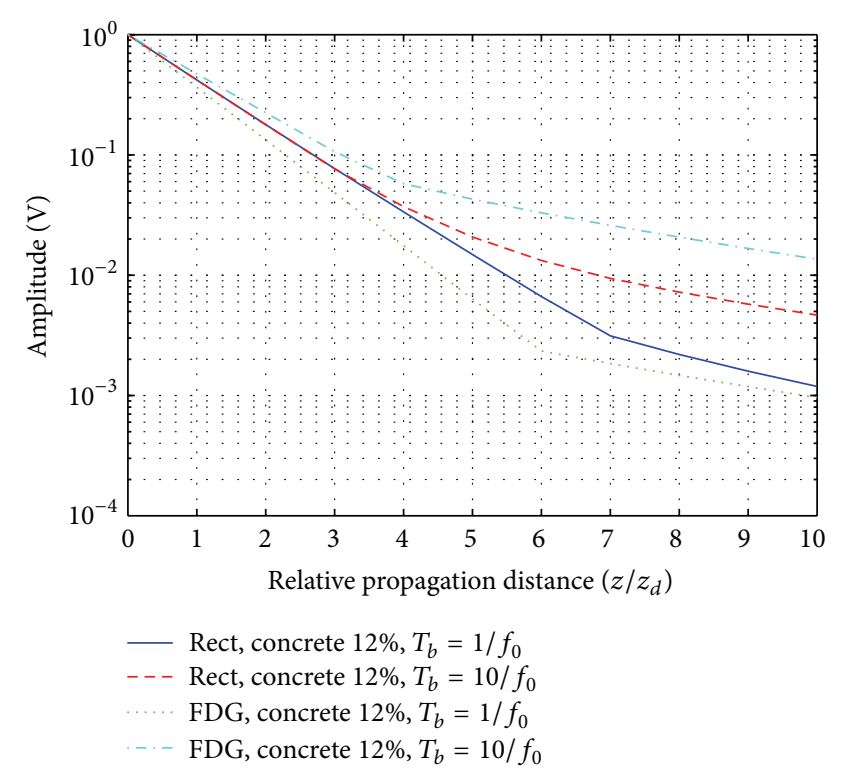

FIgURE 10: Peak amplitude as a function of propagation distance through wet concrete ( $12 \%$ moisture) for rectangular and FDG pulses of duration $T_{b}=10 / f_{0}$ and $T_{b}=1 / f_{0}$ at $f_{0}=1.5 \mathrm{GHz}$.

For simulation studies in this paper, we choose as input signals two different pulses: a rectangular pulse and first derivative of Gaussian (FDG) pulse, this latter as an approach of impulse case. The rectangular pulse is a good choice to show the pros and cons of the precursor formation. Even when widely used for UGI, in [5] we determined that the Gaussian pulse is not the best option to produce the emergence of the Brillouin and/or Sommerfield precursor with large amplitude levels due to the smoothness of its noninfinite leading edges. Because of this reason, we preferred to consider the first derivative Gaussian (FDG) that works like a modulated Gaussian pulse with a bandwidth and central frequency that are related to the time width of the Gaussian pulse, and it is related to larger amplitude level precursors due to its spread-out frequency spectrum. In case of sine carrier modulation of the baseband pulse $p(t)$, central frequency of the Gaussian pulse would equal $f_{0}$ corresponding to the carrier component. Other pulses have been analyzed as optimal [19], and they could also be applied further improving the results.

In (8) we indicate the settings used for the sequence of delta pulses and FDG:

$$
p(t)=\frac{-2(t-a)}{b^{2}} \cdot \exp \left\{-\left(\frac{t-a}{b}\right)^{2}\right\}
$$

with $(a, b)=\left(0.05 \cdot T_{b}, 0.0375 \cdot T_{b}\right)$. The expression for the rectangular pulse just involves one parameter, the pulse width, or period, $T_{b}: p(t)=\prod\left(t / T_{b}\right), 0 \leq t \leq T_{b}$.

The input pulse configuration influences the formation and evolution of the resulting precursors [3-5]. In order to show this fact, we have considered two different settings, mono- and multicycles, by choosing $T_{b}=1 / f_{0}$ and $T_{b}=$ $10 / f_{0}$ for both rectangular and FDG pulses. In Figure 3 we 


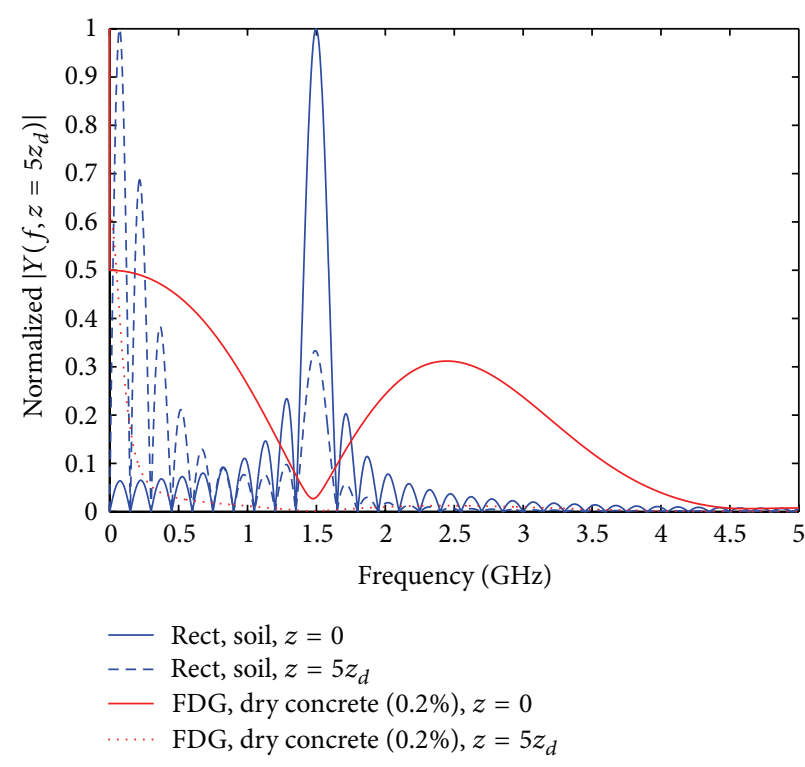

(a)

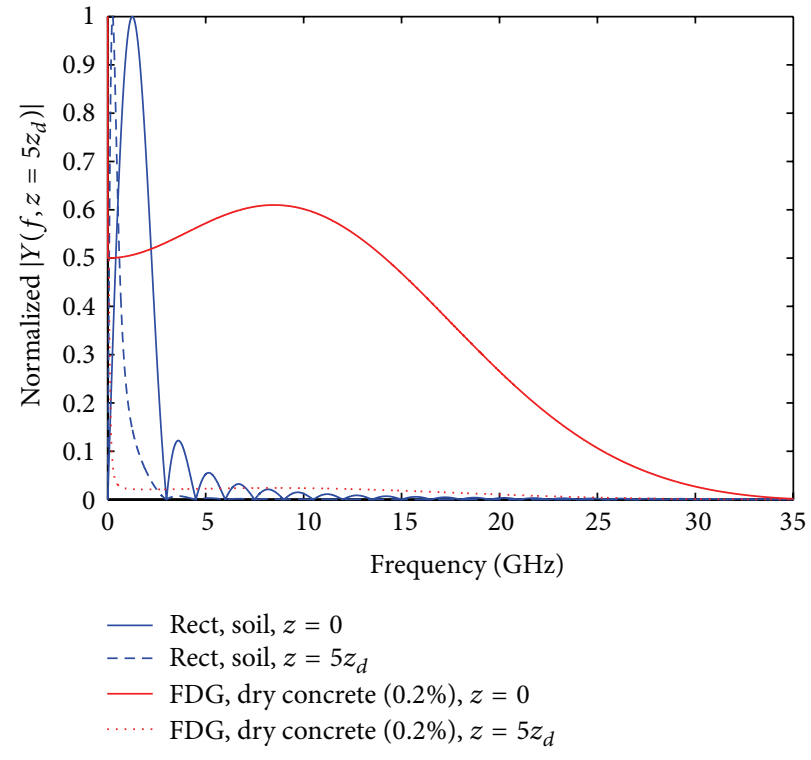

(b)

FIGURE 11: Frequency spectra for rectangular and FDG pulses after propagating through $z=4 \cdot z_{d}$ through soil and concrete at $f_{0}=1.5 \mathrm{GHz}$, with pulse duration (a) $T_{b}=10 / f_{0}$ and (b) $T_{b}=1 / f_{0}$.

show the input pulses used for this study, modulated at a carrier frequency of $f_{0}=1.5 \mathrm{GHz}$. The resulting energy contents for both types of pulses are not equal.

\section{Dynamical Evolution within the Dispersive Medium for Drude Model}

The dynamical evolution of the total field $E_{T}(z, t)$ is theoretically predicted using (1) for the Debye model and considering IMR structures of soil and concrete, for both rectangular and FDG pulses, modulated using a sine carrier frequency $f_{0}=$ $1.5 \mathrm{GHz}$. From $E_{T}(z, t)$ we can analyze the peak amplitude decay law of the propagated field which helps to determine the penetration depth that the transmitted waveform can achieve into the given medium. The electric-field intensity and the frequency spectra are also analyzed.

In the results plotted, the propagation distance is normalized with respect to the reference distance $z_{d}$ that is defined as the propagation distance $z$ at which the amplitude decay $\exp \{-\alpha(f) \cdot z\}$ of the signal travelling through a dispersive medium equals $\exp \left\{-\alpha\left(f_{0}\right) \cdot z_{d}\right\}=e^{-1}$, equivalently $1 \mathrm{~Np}$, for a given frequency value, in our case $f=f_{0}$ corresponding to the carrier component, with $\alpha(f)$ the attenuation constant of the medium.

3.1. Dynamical Evolution. In Figures 4, 5, and 6 we show the spatial and time evolution for ten propagation distances $z_{d}$ at $f_{0}=1.5 \mathrm{GHz}$ assuming the IMR model for soil, dry concrete, and wet concrete (Figure 6), respectively. In order to emphasize the weaker values for the longer propagation distances, each signal has been normalized by its peak value.

For the rectangular pulse case with $T_{b}=10 / f_{0}$, shown in Figure 4, the Brillouin precursors are observable as a superimposed wave in the leading and trailing edges of the output rectangular pulse. For the monocycle setting cases of Figures 5 and 6 , even that not so much graphically observable, we detect the appearance of the Brillouin precursor at the trailing edge of the rectangular and FDG pulses, explained by the broad lobule-shaped component indicated in Figure 5. For larger propagation distances, the Brillouin precursor becomes the predominant forerunner, and the remaining carrier frequency component is hardly observable.

For the wet concrete, the Brillouin precursor component is less noticeable, and the carrier frequency is also less attenuated, perhaps due to the larger moisture contents.

In Figures 7(a) and 7(b) we show the intensity of the normalized electric field at any given location $(z, t): I(z, t)=$ $|E(z, t)|^{2}$, estimated for a multicycle rectangular pulse and a monocycle FDG pulse through soil. In these plots, the amplitude levels present a range between 0 and 1 , the blue pixels represent locations where the intensity is at minimum, and the red pixels correspond to locations of maximum intensity [19]. In Figure 7(a), we observe that the carrier cycles extinguish after a few propagation depths and the Brillouin precursors corresponding to the leading and trailing edges of the rectangular pulse evolve following a linear track. For the monocycle case in Figure 7(b), we observe just one track due to the fact of that both precursors merge after a short propagation distance.

3.2. Peak Amplitude Decay. The maximum value of the evolving field $E_{T}\left(z=z_{k}, t\right)$ at $k$ th depth $z=z_{k}$ is plotted in Figures 8, 9, and 10 for ten normalized depths $\Delta z=$ $z / z_{d}$, for rectangular and FDG pulses with $T_{b}=1 / f_{0}$ and $T_{b}=10 / f_{0}$. These maxima values include the carrier and precursor components given that they have not been 


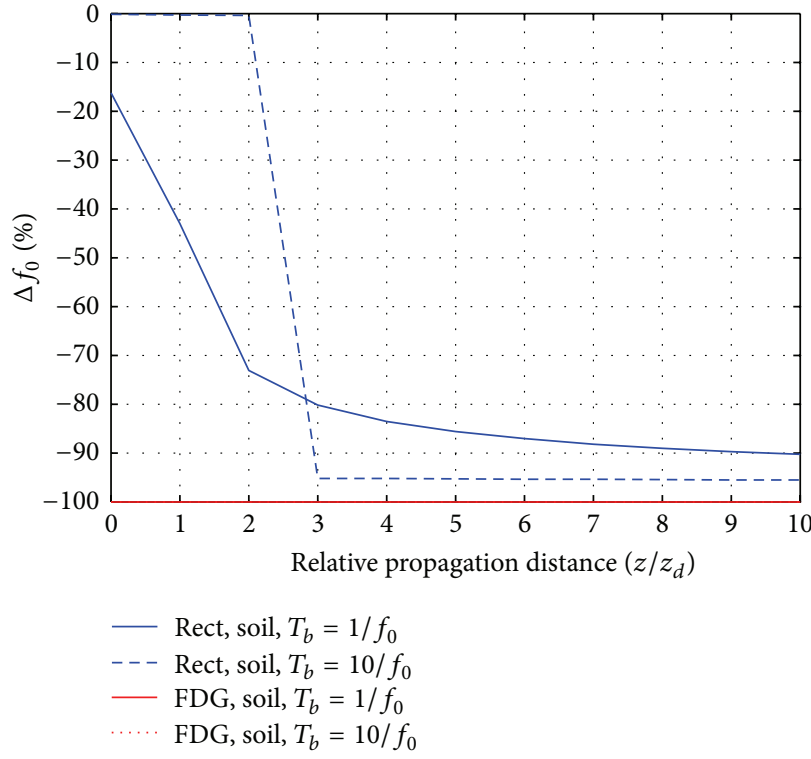

(a)

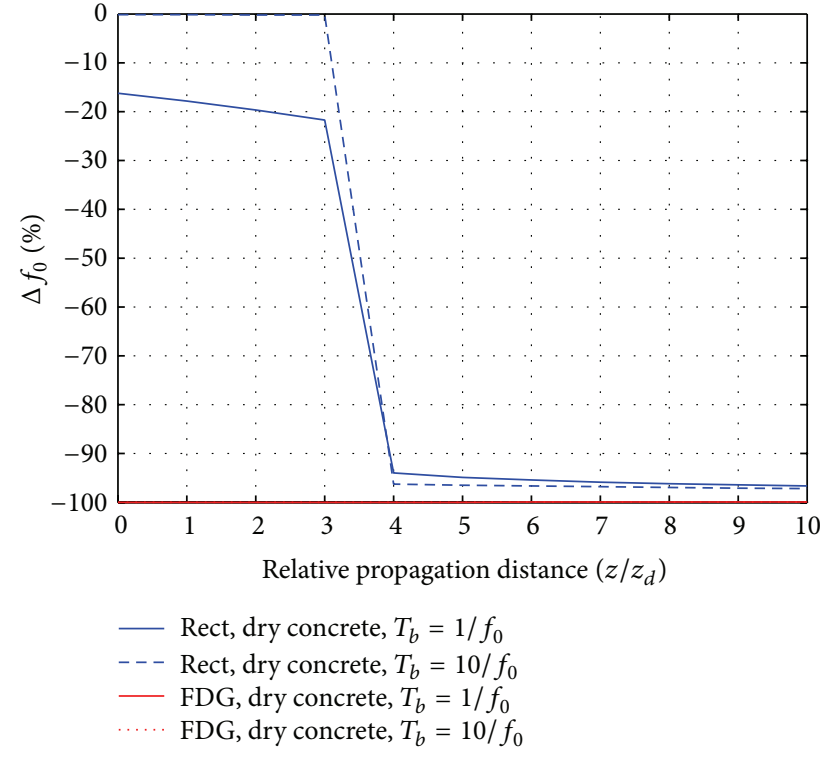

(b)

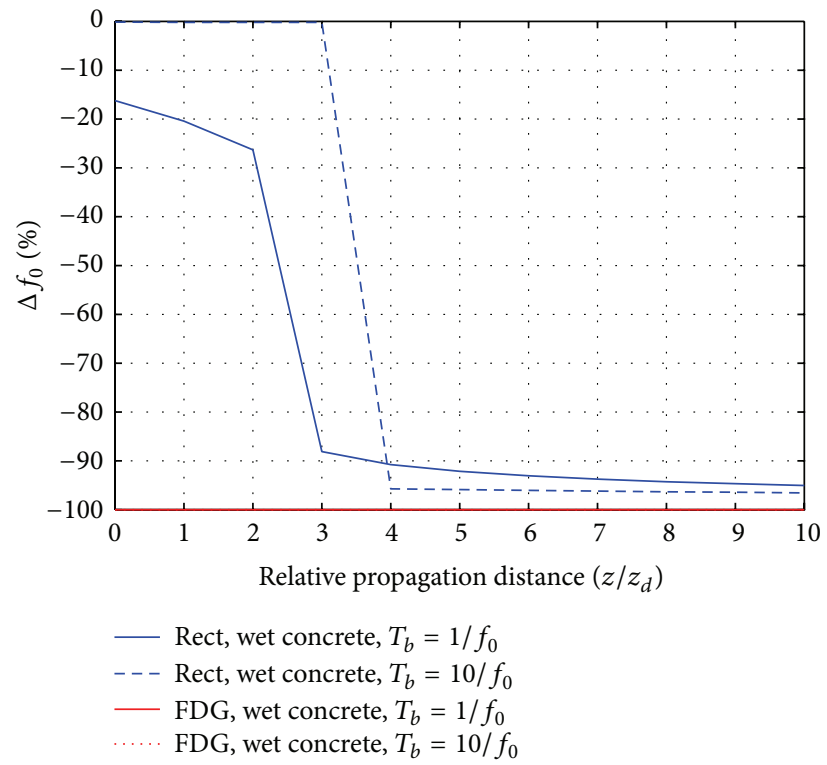

(c)

FIGURE 12: Frequency carrier shifting estimation: (a) soil, (b) dry concrete $(0.2 \%)$, and (c) wet concrete (12\%).

segregated. Figure 8 illustrates the peak decay amplitude assuming the soil model. The dry concrete case is shown in Figure 9 and wet concrete in Figure 10.

We conclude that the total propagated field $E_{T}(t, z)$ follows an algebraically decaying peak amplitude instead of an exponentially decay as given by the attenuation corresponding to the carrier frequency, with similar performance of both settings and mono- and multicycle, for the rectangular pulse; however, the pulse configuration takes a large influence for the FDG pulse even for shorter propagation distances.

We notice that for wet concrete the peak amplitude decay is slightly less attenuated, likely due to a larger presence of water.
3.3. Frequency Spectrum. In Figures 11(a) and 11(b) we show the normalized spectra for rectangular and FDG pulses after travelling through a propagation distance of $4 \cdot z_{d}$ within soil, dry, and wet concrete, respectively. We observe that the FDG pulse case undergoes a larger alteration of the spectrum shape than that of a rectangular pulse; the increasing level of the low frequencies-larger as closer to zero-is an effect of the dispersion phenomenon more remarkable for the rectangular pulse.

The percentage of downshifting with respect to the original frequency carrier $f_{0}$ was measured as $\Delta f_{0}=100 \cdot\left(f_{0}^{\prime}-\right.$ $\left.f_{0}\right) / f_{0}$ and shown in Figures 12(a), 12(b), and 12(c), only for multicycle configuration and soil medium. The rectangular 
cases offer similar results in terms of frequency carrier downshifting; however, it becomes a critical impairment for the FDG pulse.

\section{Conclusions}

Generally speaking, the results presented in this paper indicate an undeniable effect of the frequency dispersion in subsurface and through-wall imaging applications, particularly for the soil and concrete structures considered here. The propagation model has been assumed as frequency dispersive and described by the internal multireflection model, and the output waveforms indicate a more than plausible malfunction of the receiver systems which are not usually built to consider the Brillouin precursor formation.

The method herein described provides a versatile framework to theoretically analyze the phenomenon of the Brillouin precursor formation and evolution through a given medium. This tool would be also useful to observe the influence of different variables such as the type of the input signal, the carrier frequency, the pulse width, the dielectric model, the bandwidth limiting, the multipath, the filtering, and so on [3-5]. All of them condition the precursor formation.

We demonstrated the importance of the precursor fields and the further research needed in this direction. Outcomes indicate that the propagation phenomenon, derived from the analysis of Brillouin precursor waveform, cannot be neglected, especially due to the large amount of energy carried by these components. In order to take advantage of the potential benefits of the precursor fields further research must be done, both in the experimental and theoretical field.

Unfortunately, there is a gap in experimental corroboration regarding the topic of precursors and microwave frequency band. In [5] we presented experimental results achieved by measuring the precursors arising from an ad hoc prepared soil layer. Even when limited to one type of soil and one thickness, the outcomes in [5] can be considered in good agreement with the model herein proposed, concluding from both works that the SNR can be enhanced if the precursor formation is considered, thereby improving the imaging for through-wall and underground scenarios.

In [5] we did not contemplate the influence of the underlying transmission model that is introduced here as a key to further understand the precursor formation and the related results for radar imaging purposes. The combination of larger SNR, that is, larger amplitude echoes, and the transmission model could be useful in the inversion analysis through correctly interpreting the backscattered signals as a part of the internal multireflection process.

In a pulse-dependent analysis, the processing of the received signal is attached to a priori configured pulse. Then, if precursors are formed during the transmission, it could derive a malfunction of the receiver system. Differently from a pulse-dependent enunciation, a frequency domain solution provides many degrees of freedom to achieve optimal processing results derived from the possibility of selecting any input pulse, frequency bandwidth windows, number of frequency components, and so on, once the transfer function $H(z, f)$ is obtained, well experimentally from in situ scenario or from a theoretical setting. Different pulses can be analyzed in order to improve the SNR values so that weak echoes backscattered by plausible targets can be differentiated from the noise level.

Inversely, from the approach here described, an optimal pulse can be selected [19], in terms of penetration distance, amplitude decay, and time width spreading, for a specific scenario/medium in order to implement a pulse-dependent hardware.

In this paper we have defined a plausible theoretical scenario in order to show the potential advantages from considering the precursor formation. If the theoretical study is used as a way to design an optimal pulse, the practical performance of such waveform can be affected by a deviation from the assumed dielectric model and scenario parameters, as described in [3]. However, any other approach or theory can be affected by the same circumstances.

The sensitivity in Brillouin precursor formation with respect to uncertainty in the model is not as much critical as the effect of uncertainty in the dielectric properties. The precursor formation is more related to the dielectric properties of the underlying medium rather than the geometrical features of the propagation scenario. As aforementioned, the dielectric medium joint to the frequency bandwidth allocation and input pulse affects the amplitude and width of the precursor field; however, the geometrical model of the medium influences the multipath behavior and this influence is observable in the expression for the medium frequency response $H(f, z)$, and therefore it must be taken into consideration for inverse techniques in radar imaging. In this paper we have considered a scenario model different from the ideal half-space medium, and as previously indicated any other scenarios can be object of a similar analysis.

\section{Conflict of Interests}

The authors declare that there is no conflict of interests regarding the publication of this paper.

\section{Acknowledgments}

This work was supported by Galician Regional Government under Project EM2012/138. The authors would also like to thank the support received from the New Mexico State University (NMSU), Las Cruces, NM, USA.

\section{References}

[1] K. E. Oughstun, Electromagnetic and Optical Pulse Propagation, vol. 2, Springer, New York, NY, USA, 2009.

[2] K. E. Oughstun, "Dynamical evolution of the Brillouin precursor in Rocard-Powles-Debye model dielectrics," IEEE Transactions on Antennas and Propagation, vol. 53, no. 5, pp. 1582-1590, 2005.

[3] A. V. Alejos, M. Dawood, and H. U. Mohammed, "Analysis of brillouin precursor propagation through foliage for digital sequences of pulses," IEEE Geoscience and Remote Sensing Letters, vol. 8, no. 1, pp. 59-63, 2011. 
[4] A. V. Alejos and M. Dawood, "Estimation of power extinction factor in presence of brillouin precursor formation through dispersive media," Journal of Electromagnetic Waves and Applications, vol. 25, no. 4, pp. 455-465, 2011.

[5] H. U. R. Mohammed, M. Dawood, and A. V. Alejos, "Experimental detection and characterization of Brillouin precursor through loamy soil at microwave frequencies," IEEE Transactions on Geoscience and Remote Sensing, vol. 50, no. 2, pp. 436445, 2012.

[6] H. Mohammed, M. Dawood, and A. Vázquez Alejos, "Experimental detection of Brillouin precursors through tap water at microwave frequencies," IET Electronics Letters, vol. 42, no. 25, pp. 1645-1647, 2010.

[7] W. Honcharenko and H. L. Bertoni, "Transmission and reflection characteristics at concrete block walls in the UHF bands proposed for future PCS," IEEE Transactions on Antennas and Propagation, vol. 42, no. 2, pp. 232-239, 1994.

[8] C. Thajudeen, A. Hoorfar, F. Ahmad, and T. Dogaru, "Measured complex permittivity of walls with different hydration levels and the effect on power estimation of twri target returns," Progress In Electromagnetics Research B, no. 30, pp. 177-199, 2011.

[9] O. Landron, M. Feuerstein, and T. Rappaport, "In situ microwave reflection coefficient measurements for the rough exterior wall surfaces," in Proceedings of the 43rd IEEE Vehicular Technology Conference, pp. 77-80, May 1993.

[10] A. Ogunsola, U. Reggiani, and L. Sandrolini, "Modelling shielding properties of concrete," in Proceedings of the 17th International Zurich Symposium on Electromagnetic Compatibility, pp. 34-37, March 2006.

[11] A. Ogunsola, U. Reggiani, and L. Sandrolini, "Shielding properties of conductive concrete against transient electromagnetic disturbances," in Proceedings of the IEEE International Conference on Microwaves, Communications, Antennas and Electronics Systems (COMCAS '09), Tel-Aviv, Israel, November 2009.

[12] J. Raymond Luebbers, Tom Uno, and Ken Kumagai, "Comments on 'pulse propagation in a linear, causally dispersive medium,' Proceedings of the IEEE, vol. 81, no. 4, pp. 631-639, 1993.

[13] J. L. Young and R. O. Nelson, "A summary and systematic analysis of FDTD algorithms for linearly dispersive media," IEEE Antennas and Propagation Magazine, vol. 43, no. 1, pp. 61$77,2001$.

[14] S. W. Samn, "Modeling dispersive dielectric media in FDTD: a systematic approach," IEEE Transactions on Antennas and Propagation, vol. 53, no. 10, pp. 3367-3373, 2005.

[15] R. Albanese, J. Penn, and R. Medina, "Short-rise-time microwave pulse propagation through dispersive biological media," Journal of the Optical Society of America A, vol. 6, no. 9, pp. 1441-1446, 1989.

[16] P. M. Jordan and A. Puri, "Digital signal propagation in dispersive media," Journal of Applied Physics, vol. 85, no. 3, pp. 1273-1282, 1999.

[17] C. A. Balanis, Advanced Engineering Electromagnetics, John Willey and Sons, 1989.

[18] K. R. Demarest, Engineering Electromagnetics, Prentice Hall, 1st edition, 1997.

[19] A. V. Alejos, M. Dawood, and H. U. R. Mohammed, "Empirical pseudo-optimal waveform design for dispersive propagation through loamy soil," IEEE Geoscience and Remote Sensing Letters, vol. 5, no. 9, pp. 953-957, 2012. 

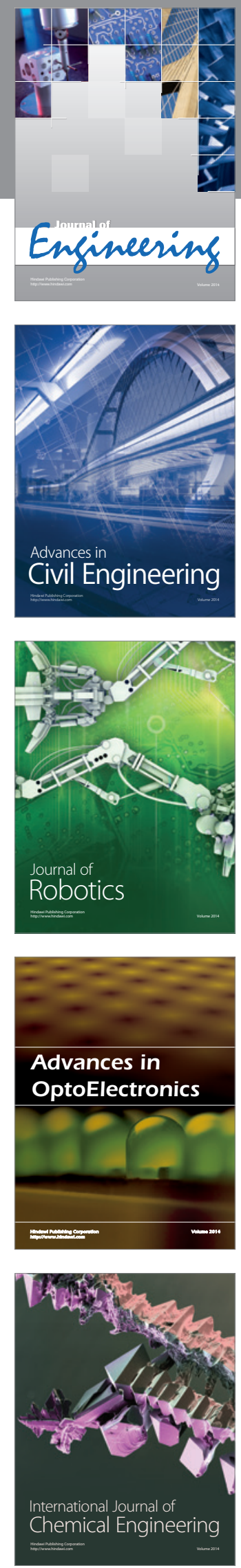

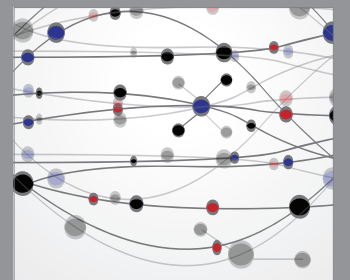

The Scientific World Journal
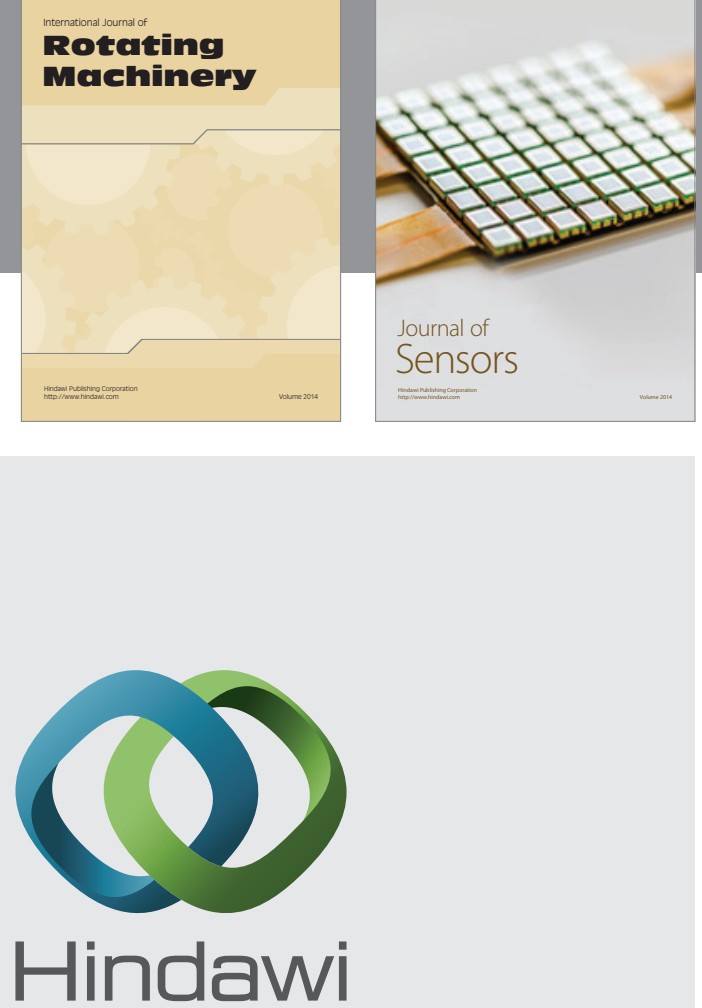

Submit your manuscripts at http://www.hindawi.com
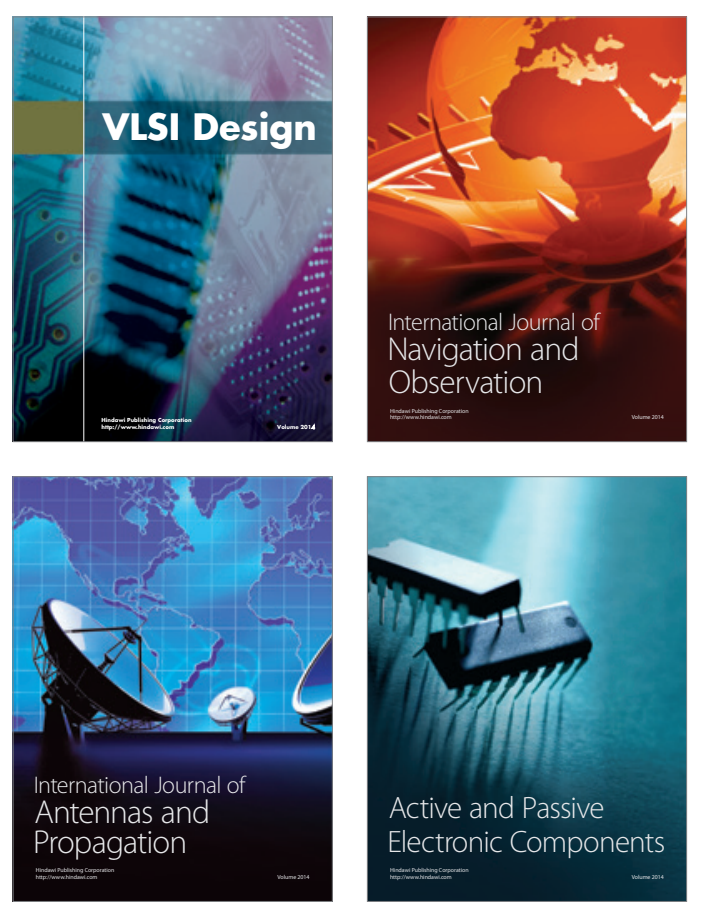
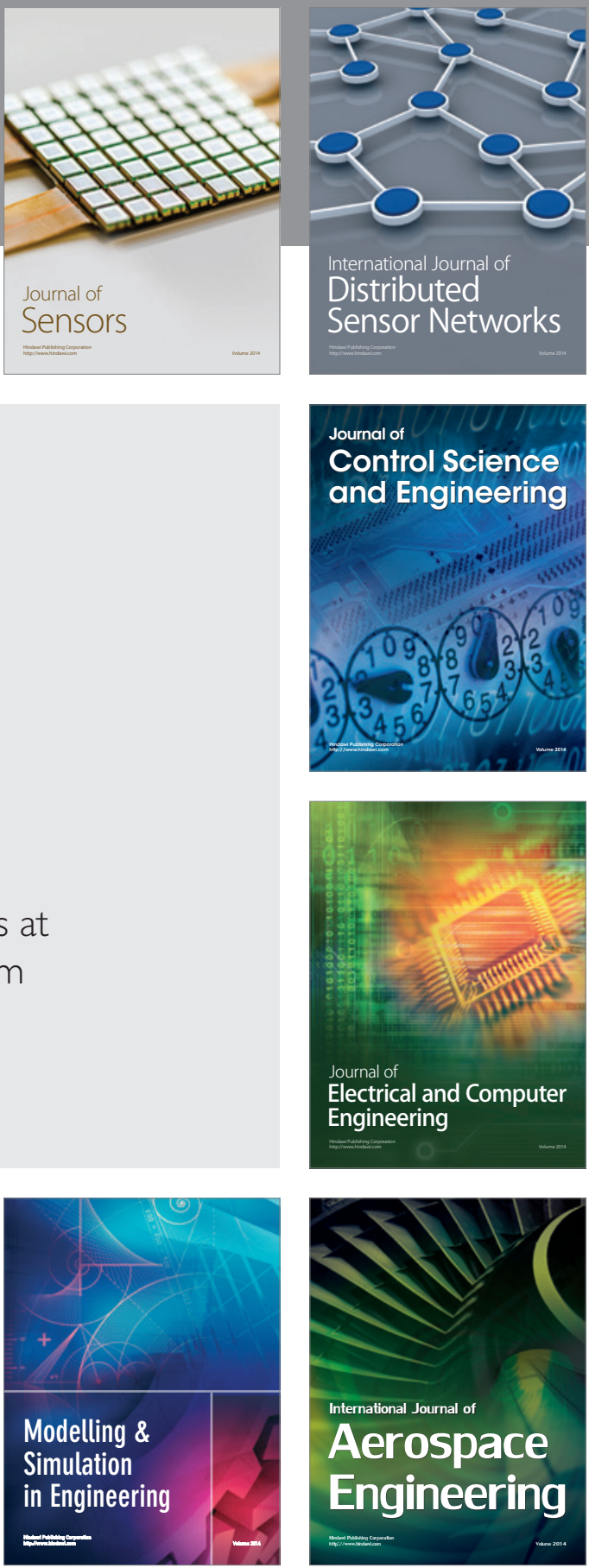

Journal of

Control Science

and Engineering
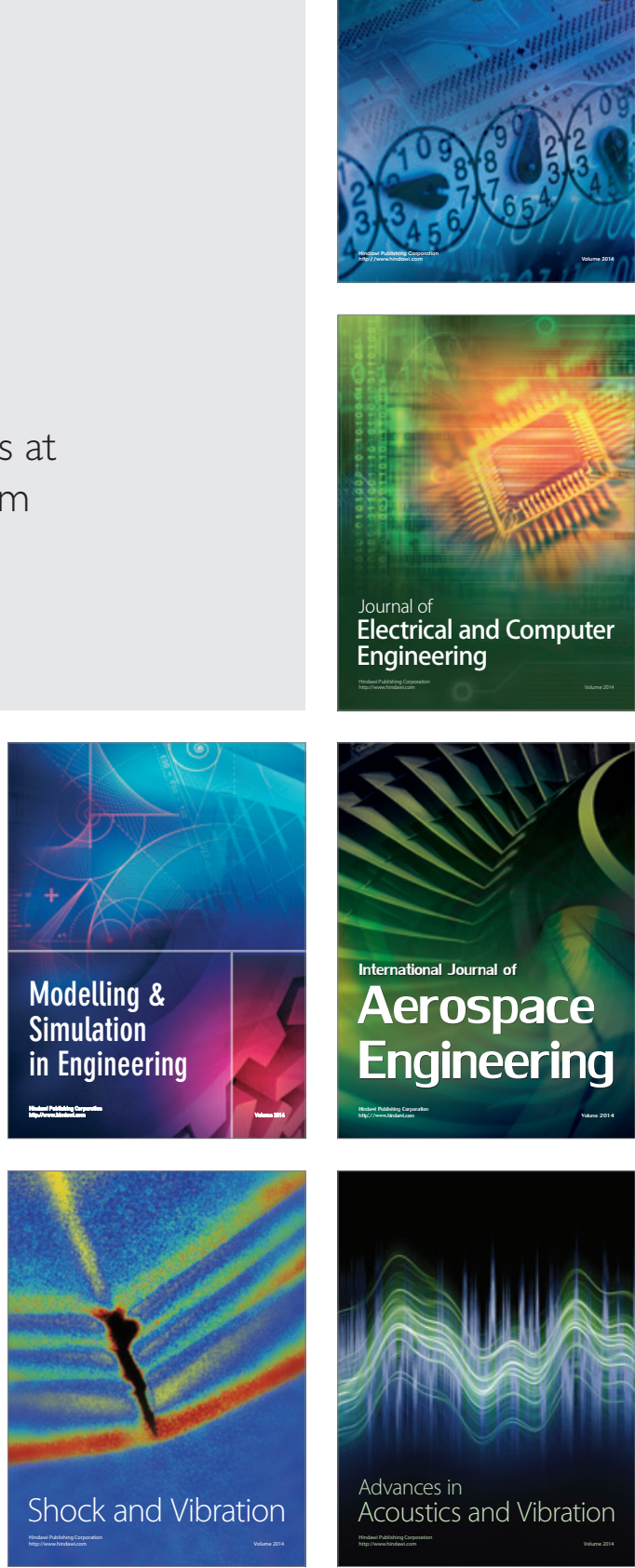